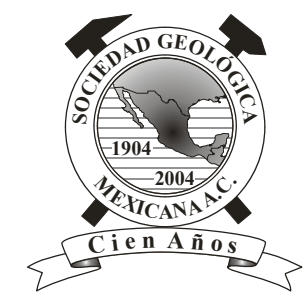

\title{
Flor de una nueva especie de Lunania Hook. (Salicaceae sensu lato - Samydeae) incluida en ámbar del Mioceno de Simojovel de Allende, Chiapas, México
}

\author{
Ana L. Hernández-Damián ${ }^{1, *}$, Laura Calvillo-Canadel1², Sergio R. S. Cevallos-Ferriz ${ }^{3}$ \\ ${ }^{1}$ Posgrado en Ciencias Biológicas, Universidad Nacional Autónoma de México, Ciudad Universitaria, C.P. 04510, \\ Coyoacán, CDMX, México. \\ ${ }^{2}$ Departamento de Botánica, Instituto de Biología, Universidad Nacional Autónoma de México, Ciudad Universitaria, Circuito \\ Exterior, Coyoacán, 04510, CDMX, México. \\ ${ }^{3}$ Departamento de Paleontología, Instituto de Geología, Universidad Nacional Autónoma de México, Ciudad Universitaria, Circuito \\ de la Investigación Científica, Coyoacán, 04510, CDMX, México. \\ *hdez_damian@ciencias.unam.mx,scrscfpb@icloud.com
}

\section{Resumen}

Los depósitos de ámbar de Simojovel de Allende, Chiapas, México constituyen uno de los más importantes del Mioceno a nivel mundial; sin embargo, los estudios paleontológicos reportados en la zona son escasos, en especial los relacionados con las plantas. En este trabajo se amplía el registro taxonómico de las angiospermas en la localidad fosilífera, al confirmar la presencia de Salicaceae sensu lato con base en una flor bisexual, inconspicua, pedicelada, con dos cicatrices de bractéolas en la base, la presencia de 3 sépalos cóncavos, pétalos ausentes, disco hipógino, 15 estambres libres y distribuidos en dos verticilos (el externo más largo que el interno) y ovario súpero. Estos caracteres sugieren que se trata de una especie extinta del género Lunania, al presentar una mezcla de caracteres de dos especies: L. parviflora y L. mexicana. La presencia de Lunania en el Mioceno de México, constituye evidencia física de la larga historia del linaje en el país apoyando, a su vez, la presencia de una selva tropical.

Palabras clave: Ámbar, flor fósil, Simojovel de Allende, Lunania, Salicaceae.

\begin{abstract}
Amber from Simojovel de Allende, Chiapas, Mexico, is one of the most important Miocene deposits worldwide; however paleontological studies based on material from the area are scarce, especially those related to plants. This taxonomic work confirms the presence of Salicaceae sensu lato in the deposit based on an inconspicuous bisexual flower, with a pedicell having two scars on its base suggesting the position of two bracteoles, hipogenous disk, 3 concave sepals, lack of petals, 15 free stamens distributed in two cycles (the longest ones are external), and superior ovary. These characters suggest that this is an extinct species of the genus Lunania, due to the fact that it has a suite of characters of the two extant species L. parviflora and L. mexicana. The presence of Lunania in the Miocene of Mexico, is physical evidence of the long history of the lineage in the country, and supports that at that time a tropical forest was developed in Simojovel de Allende.
\end{abstract}

Keywords: Amber, fossil flower, Simojovel de Allende, Lunania, Salicaceae. 


\section{Introducción}

Simojovel de Allende, Chiapas, constituye uno de los depósitos de ámbar más importantes del Cenozoico (Solórzano-Kraemer, 2007, 2010). En él se han conservado de manera excepcional organismos completos o fragmentos de ellos, como las plantas, cuyo registro fósil es relativamente más escaso en la zona, en comparación con otros grupos biológicos, como el de los insectos (Avendaño-Gil et al., 2012). Dentro del registro de plantas fósiles el grupo de las angiospermas es el mejor representado, en su mayoría por flores. Éstas constituyen un caso especial, ya que se tratan de órganos delicados y efímeros, con un alto potencial sistemático, al ser fácilmente observables (Bhattacharya, 2005) y más consistentes en sus caracteres, en comparación con órganos vegetativos que al ser sometidos a la presión del medio ambiente fluctuante (Sivarajan, 1991).

Las flores fósiles reportadas en los depósitos de ámbar de Simojovel de Allende se han identificado como miembros de distintas familias. Por ejemplo: Fabaceae con Hymenaeae mexicana (Poinar y Brown, 2002) y H. allendis (CalvilloCanadell et al., 2010), reconocidas hasta ahora como las dos especies extintas que dieron origen al ámbar; además de Anacardiaceae representada por Tapirira durhamii (Miranda, 1963), Arecaceae por Socratea brownii (Poinar, 2002), Colpothrinax chiapensis (Chambers et al., 2012) y Meliaceae por Swietenia miocenica (Castañeda-Posadas y Cevallos-Ferriz, 2007). En este trabajo se amplía el registro taxonómico de las angiospermas con una nueva especie extinta de Salicaceae sensu lato (s.l.) perteneciente al género Lunania.

Salicaceae sensu stricto (s.s.) está constituida por dos géneros Salix y Populus, sin embargo, la actual circunscripción de la familia propuesta por Chase et al. (2002) a través de un análisis filogenético con datos moleculares ( $\mathrm{rbcL}$ ) de Flacourtiaceae, sugiere que la mayoría de los géneros incluidos en Flacourtiaceae forman parte de Salicaceae, ampliando la circunscripción de la familia a $c a .54-55$ géneros y dividiéndola en nueve tribus: Saliceae, Flacourtieae, Scyphostegieae, Homalieae, Scolopieae, Prockieae, Abatieae, Bembicieae, y Samydeae. Esta última es considerada por algunos taxónomos como una familia (Samydaceae), dejando al resto como Salicaceae sensu medio, pero este enfoque no ha sido ampliamente aceptado (Alford y Belyaeva, 2009).

La tribu Samydeae se encuentra constituida por 12 géneros, incluido Lunania Hook (Chase et al., 2002). Lunania es un género neotropical, constituido por $c a .14$ especies (Hooker, 1844; Robyns, 1968; Chase et al., 2002) de árboles y arbustos, con flores pequeñas, bisexuales, en racimos axilares o terminales y sostenidas por dos bractéolas. Sin embargo, las bases de datos recientes como The Plant List (2013) sólo reconoce a tres especies: $L$. parviflora, L. mexicana y L. bauchii. Este trabajo representa al primer registro fósil del género y está basado en una flor.

\section{Materiales y métodos}

\subsection{Localidad}

El material fósil fue colectado en el municipio de Simojovel de Allende en el Estado de Chiapas (17 $08^{\prime} 19^{\prime \prime} \mathrm{N}$ y $92^{\circ} 43^{\prime} 00$ 'O) a una altitud de $600 \mathrm{msnm}$ (Figura 1). El área está limitada al Norte por los municipios de Huitiupán, Sabanilla y Tila; al Este por el municipio de Chilón; al Sur por los municipios de Pantheló, Chalchihuitán y el Bosque; al Oeste por Jitotol y Nuevo Pueblo Solistahuacán (Centro Estatal de Estudios Municipales [CEEM], 1988). El clima que presenta esta región es cálido-subhúmedo, con lluvias veraniegas en abundancia, y semicálido-húmedo con lluvias todo el año. Los meses más cálidos son abril y mayo.

\subsection{Estratigrafía}

Böse (1905) realizó una síntesis geográfica y fisiográfica de la zona, dividiendo la secuencia estratigráfica cenozoica en: división Eoceno, división Simojovel (Oligoceno), división Tenejaoa (Plioceno) y división Cuaternario. Posteriormente, Frost y Langenheim (1974) dividieron al Grupo Simojovel en Arenisca La Trinidad, Arenisca Rancho Berlín y Formación La Quinta; ésta última es donde se encuentra asociado el ámbar, aparentemente incluido en una secuencia marina del Oligoceno como lo sugieren foraminíferos planctónicos identificados, como Globigerina ciperoensis Bolli y Globerotalia kugleri Bolli reportados por los mismos autores. Sin embargo, Grimaldi (1996) mencionó que muchos depósitos de ámbar se encuentran asociados con lignitas, pizarras friables y arcillas deltaicas del Mioceno temprano. La última interpretación es consistente con estudios de nanoplanctón localizados en sedimentos de las biozonas N3 y N4, a las que se les ha asignado una edad radiométrica con rangos de 22.5 26.0 Ma (Berggren y Van Couvering, 1974). Sin embargo, Solórzano-Kraemer (2010) asigna el ámbar de Simojovel de Allende al Mioceno medio (15-20 Ma), debido al parecido que presenta con los depósitos de ámbar de la República Dominicana, incluyendo la presencia de lechos de lignita, un similar registro fósil de insectos ( $40 \%$ y y su origen botánico por especies extintas de Hymenaea. Sin embargo, Vega et al. (2009) usando ${ }^{87} \mathrm{Sr} /{ }^{86} \mathrm{Sr}$ en conchas bien preservadas de Turbinella maya de Los Pocitos, una localidad muy cercana a Simojovel, establecen una edad de $23 \mathrm{Ma}$.

\subsection{Especímenes}

El material descrito e identificado es una flor preservada en ámbar del Mioceno de Simojovel de Allende, perteneciente a la colección del Museo de Paleontología "Eliseo Palacios Aguilera", de la Secretaría de Medio Ambiente, Vivienda e Historia Natural (SEMAVIHN), de Tuxtla Gutiérrez, Chiapas, con número de catálogo 5963-IHNFG-2919 (IHNFG-Instituto de Historia Natural-Fósiles Geográficos). 

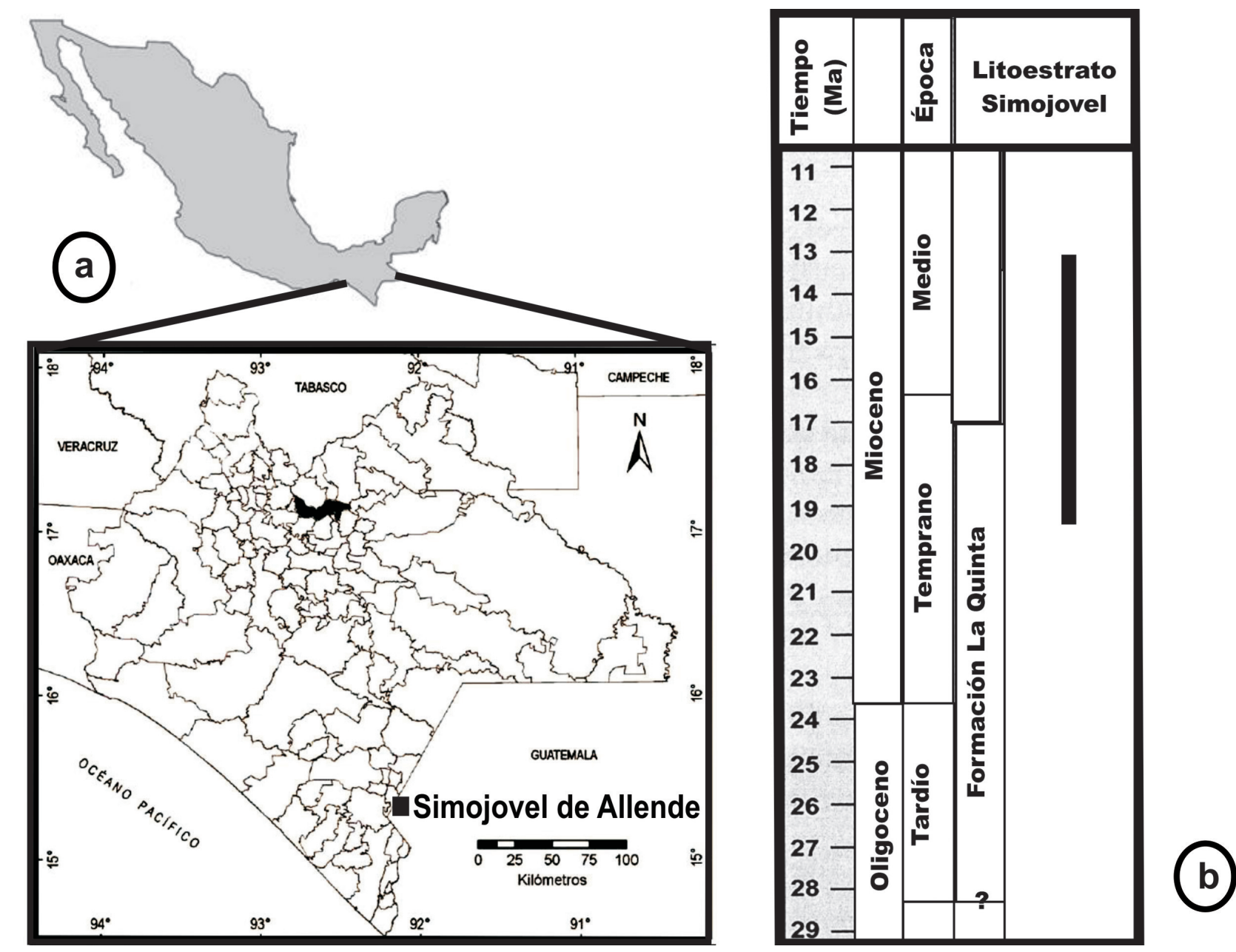

Figura 1. (a) Localización de los depósitos de ámbar de Simojovel de Allende. (b) Edad de los depósitos (Tomado y modificado de Solórzano-Kraemer, 2010).

La flor fósil, inicialmente fue descrita e identificada por Gómez-Bravo (2005) como un miembro de Salicaceae. Para llevar a cabo su reexaminación, se usó la clave electrónica para los géneros de Flacourtiaceae de Alford (2012), y se ingresaron los siguientes caracteres: sexo de la flor (17), perianto (18), arreglo del perianto (22), largo de los sépalos (23), posición del disco (24), forma de la antera (25), unión de los estambres (39), estaminodios (40) y arreglo de los estambres (42). Así mismo, se llevó a cabo una revisión bibliográfica basada en Sleumer (1980), Alford (2003), Robyns (1968), Wild (1960) y Yang y Zmarzty (2007), en las que se buscaron los taxones actuales con mayor similitud morfológica con la flor fósil. Una vez identificados, se buscaron ejemplares con estructuras reproductoras en los herbarios MEXU y MEX:TUX, para ser examinados y constatar su parecido.

\section{Resultados}

\subsection{Descripción sistemática}

Familia: Salicaceae Mirbel, 1815

Tribu: Samydeae (Vent.) Dumort, 1829

Género: Lunania Hooker, 1844

\section{Lunania floresi Hernandez-Damián, Calvillo-Canadell, Cevallos-Ferriz sp. nov.}

Diagnosis. Flor bisexual; inconspicua, pedicelada, 2 bractéolas; sépalos 3, parcialmente fusionados hacia la base, cóncavos; pétalos ausentes; disco hipógino, lobado; estambres 15 , distribuidos en dos verticilos (el externo más largo que el interno), anteras bitecas, biloculares, basifijas, ovado-elípticas, ligeramente apendiculares; ovario súpero, pubescente y 3 estilos cortos.

Descripción. Flor bisexual de $4.0 \mathrm{~mm} \times 2.0 \mathrm{~mm}$, inconspicua, actinomorfa, pedicelo de $1.0 \mathrm{~mm} \times 0.2 \mathrm{~mm}$, punctato, subglabro, con 2 cicatrices de bractéolas en la base; sépalos 3 (Figura 2, a, b c), parcialmente fusionados hacia la base, cóncavos, $c a .1 .5 \mathrm{~mm} \times 0.5 \mathrm{~mm}$, venación reticulada, punctato, subglabros en haz y envés; pétalos 0 ; disco hipógino, lobado, lóbulos cimbiformes (Figura 2, c); estambres 15 , libres, distribuidos en dos verticilos, estambres 
del verticilo exterior de $c a .1 .5 \mathrm{~mm} \times 0.1 \mathrm{~mm}$, estambres del verticilo interior de $c a .1 .0 \mathrm{~mm} \times 0.1 \mathrm{~mm}$, filiformes (Figura 2, d); anteras bitecas, biloculares, ovado-elípticas, basifijas $0.5 \mathrm{~mm} \times 0.4 \mathrm{~mm}$, ligeramente apendiculares (Figura 2, e); ovario súpero, ovado y pubescente; 3 estilos terminales y cortos (Figura 2, f).
Epíteto específico. El nombre hace referencia a la Dra. Hilda Flores Olvera, botánica mexicana que ha tenido un enorme interés en el desarrollo de la paleobotánica en el país.

Ejemplar examinado. 5963-IHNFG-2919 (IHNFGInstituto de Historia Natural-Fósiles Geográficos).

Repositorio. Colección del Museo de Paleontología
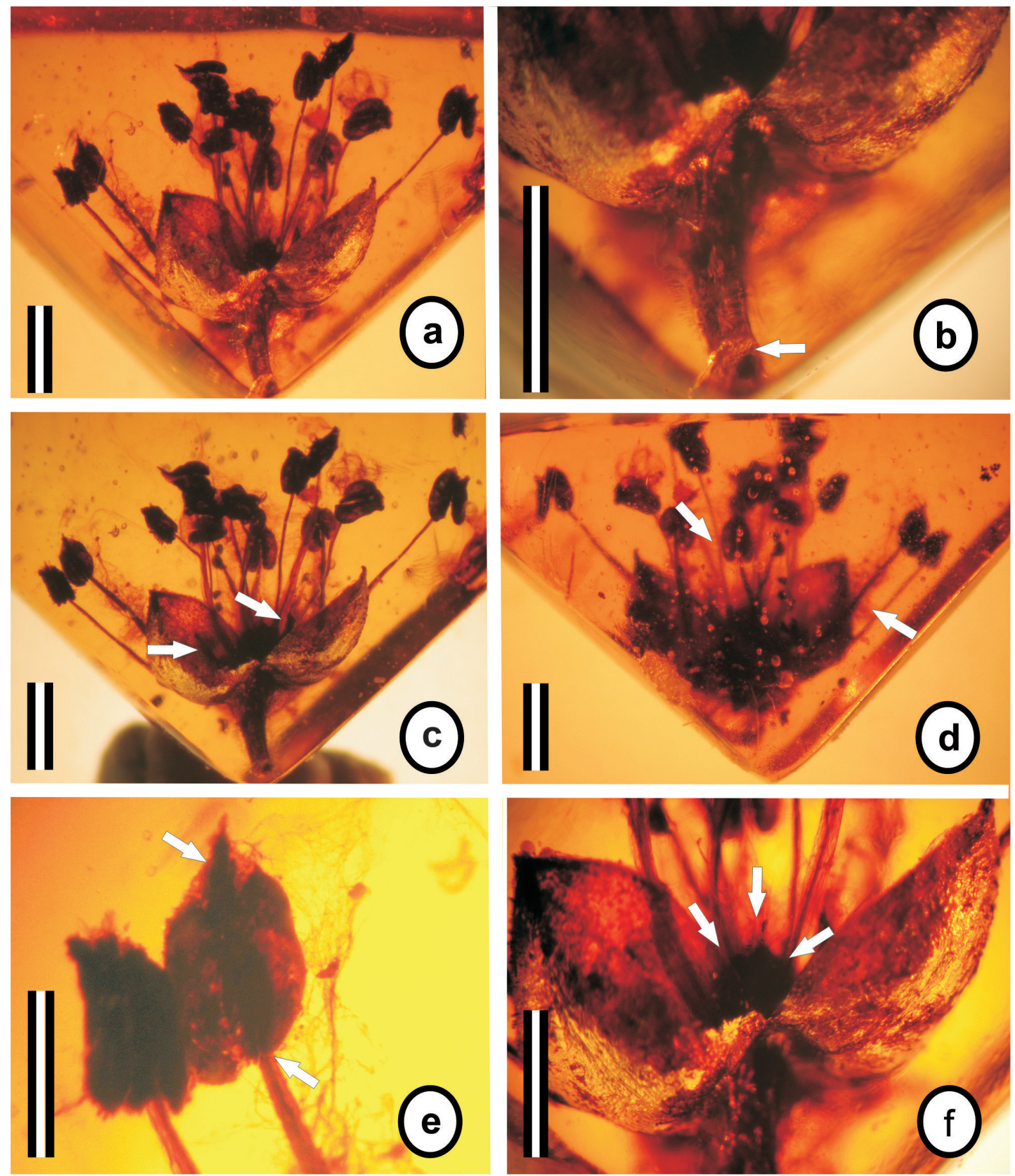

Figura 2. Lunania floresi sp. nov. (a) Flor bisexual, actinomorfa, pedicelada, con 3 sépalos cóncavos (escala $=1.0 \mathrm{~mm}$ ). (b) Pedicelo. Flecha señalando 2 cicatrices de bractéolas (escala $=1.0 \mathrm{~mm}$ ). (c) Flecha señalando disco hipógino con lóbulos (escala $=1.0 \mathrm{~mm}$ ). (d) Flecha señalando estambres libres distribuidos en dos verticilos (escala $=1.0 \mathrm{~mm}$ ). (e) Flecha señalando antera basifija y apendicular (escala $=0.5 \mathrm{~mm}$ ). (f) Ovario súpero, pubescente. Flecha señalando tres estilos cortos (escala $=0.5 \mathrm{~mm})$. 
"Eliseo Palacios Aguilera", de la Secretaria de Medio Ambiente, Vivienda e Historia Natural (SEMAVIHN) de Tuxtla Gutiérrez, Chiapas.

\section{Discusión y conclusión}

Un nuevo miembro de la familia Salicaceae s.l. fue identificado en el ámbar del Mioceno de Simojovel de Allende, con base en una flor bisexual, pedicelada e inconspicua, que presenta 2 cicatrices de bractéolas, 3 sépalos, pétalos ausentes, disco hipógino, lobado y ovario súpero con 3 estilos. Estos caracteres han permitido ubicarla en el género Lunania. Al comparar la morfología de la flor fósil con la de especies actuales de Lunania se reconoció un mayor parecido con L. mexicana y L. parviflora (Tabla 1), destacando el tamaño de los filamentos del fósil con el de $L$. mexicana $(\sim 1.5 \mathrm{~mm})$, así como las anteras apiculadas presentes en L. parviflora (Vázquez-Torres et al., 2010; Robyns, 1968). A pesar del enorme parecido que presentan entre ellas existen también diferencias importantes como el número de estambres (15 vs. 10 - 12), la vestidura de la superficie del ovario pubescente en el fósil, subglabro en $L$. mexicana y tomentoso en L. parviflora y el tamaño de los estilos (0.3 mm vs. $1.0 \mathrm{~mm}$ ) (Figura 3). Las observaciones y comparaciones morfológicas realizadas indican que el fósil presenta un mosaico de caracteres entre L. mexicana y L. parviflora así como caracteres únicos, lo que sugiere que se trata de una especie nueva y extinta de Lunania, constituyendo el primer registro del género y de Salicaceae para la zona.

En México el registro fósil de la familia se encuentra constituido por hojas de Populus, provenientes del Eoceno de Nuevo León (Pérez-Maussan, 2013), así como del Oligoceno de Puebla, donde además se ha reportado Salix (Ramírez y Cevallos-Ferriz, 2000). La presencia de Lunania en el Mioceno de Chiapas, amplía el registro de la familia espacial y temporalmente en México, esto en su actual circunscripción al haber desaparecido la familia Flacourtiaceae (Chase et al., 2002).

El registro fósil de Salicaceae se extiende desde el Paleoceno, con hojas de Populus del norte de los Estados Unidos de Norte América (Wing, 1981; Collinson, 1992), aunque el primer registro inequívoco de la familia está representado por frutos y hojas del Eoceno, de la Formación Green River, Utah y Colorado (Manchester et al, 1986; Boucher et al., 2003; Manchester et al., 2006). Sin embargo, cabe señalar que el registro fósil de Salicaceae s.l. está constituido por órganos vegetativos y reproductivos de distintas edades, que incluyen por ejemplo maderas de Aphloioxylon del Paleoceno de Groenlandia (Mathiesen, 1961), Flacourtioxylon del Eoceno de Europa (Greguss,1969; Trivedi y Srivastava, 1986; Crawley, 2001), Homalioxylon del Mioceno de Europa y Asia (Prakash y Tripathi, 1972; Bande, 1974; Gottwald, 1997), Hydnocarpoxylon del Mioceno-Plioceno de Asia (Bande y Khatri, 1980; Awasthi y Srivastava, 1989); hojas de Idesia del Mioceno de California (MacGinitie, 1937), semillas y frutos de Poliothyrsis del Oligoceno-Mioceno de Alemania (Mai, 1980), así como Saxifragispermum del Eoceno de Inglaterra (Chandler, 1961) y Oregon (Manchester, 1994). Así mismo, se han reportado casos excepcionales de órganos en conexión orgánica como lo demuestra Pseudosalix handleyi del Eoceno de Estados Unidos (Colorado) (Boucher et al., 2003).

Actualmente en México el género Lunania se encuentra

Tabla 1. Comparación de caracteres florales entre Lumania floresi, L. mexicana y L. parviflora (Sleumer, 1980; Vázquez-Torres et al., 2010; Robyns, 1968).

\begin{tabular}{lccc}
\hline & Lumania floresi & L. mexicana & L. parviflora \\
\hline Longitud del pedicelo & $1.0 \mathrm{~mm}$ & $1.0-1.5 \mathrm{~mm}$ & $1.5-2.0 \mathrm{~mm}$ \\
Bractéolas & cicatrices $(2)$ & 2 & 2 \\
Número de sépalos & 3 & $(2) 3$ & $(2) 3$ \\
Forma de los sépalos & cóncavo (globoso) & cóncavo (globoso) & cóncavo (globoso) \\
Superficie de los sépalos & glabros & glabros & puberulento \\
Disco & hipógino & hipógino & hipógino \\
Forma de los lóbulos del disco & cimbiformes & elípticos & elípticos \\
Número de estambres & 15 & 10 & $10-12$ \\
Filamentos & $1.5-1.0 \mathrm{~mm}$ & $1.5-2.0 \mathrm{~mm}$ & $0.2-0.3 \mathrm{~mm}$ \\
Lóculos de las anteras & bilocular & bilocular & bilocular \\
Longitud de las anteras & $0.5 \mathrm{~mm}$ & $0.7 \mathrm{~mm}$ & $1.5 \mathrm{~mm}$ \\
Anteras apiculadas & presentes & ausentes & presentes \\
Posición del ovario & súpero & súpero & súpero \\
Forma del ovario & ovado & ovado & ovado \\
Superficie del ovario & pubescente & subglabro & tomentoso \\
Estilo & 3 & 3 & 3 \\
Longitud del estilo & $0.3 \mathrm{~mm}$ & $1.0 \mathrm{~mm}$ & $1.0 \mathrm{~mm}$ \\
\hline & & & \\
\hline & 3 & 3 & \\
\hline
\end{tabular}



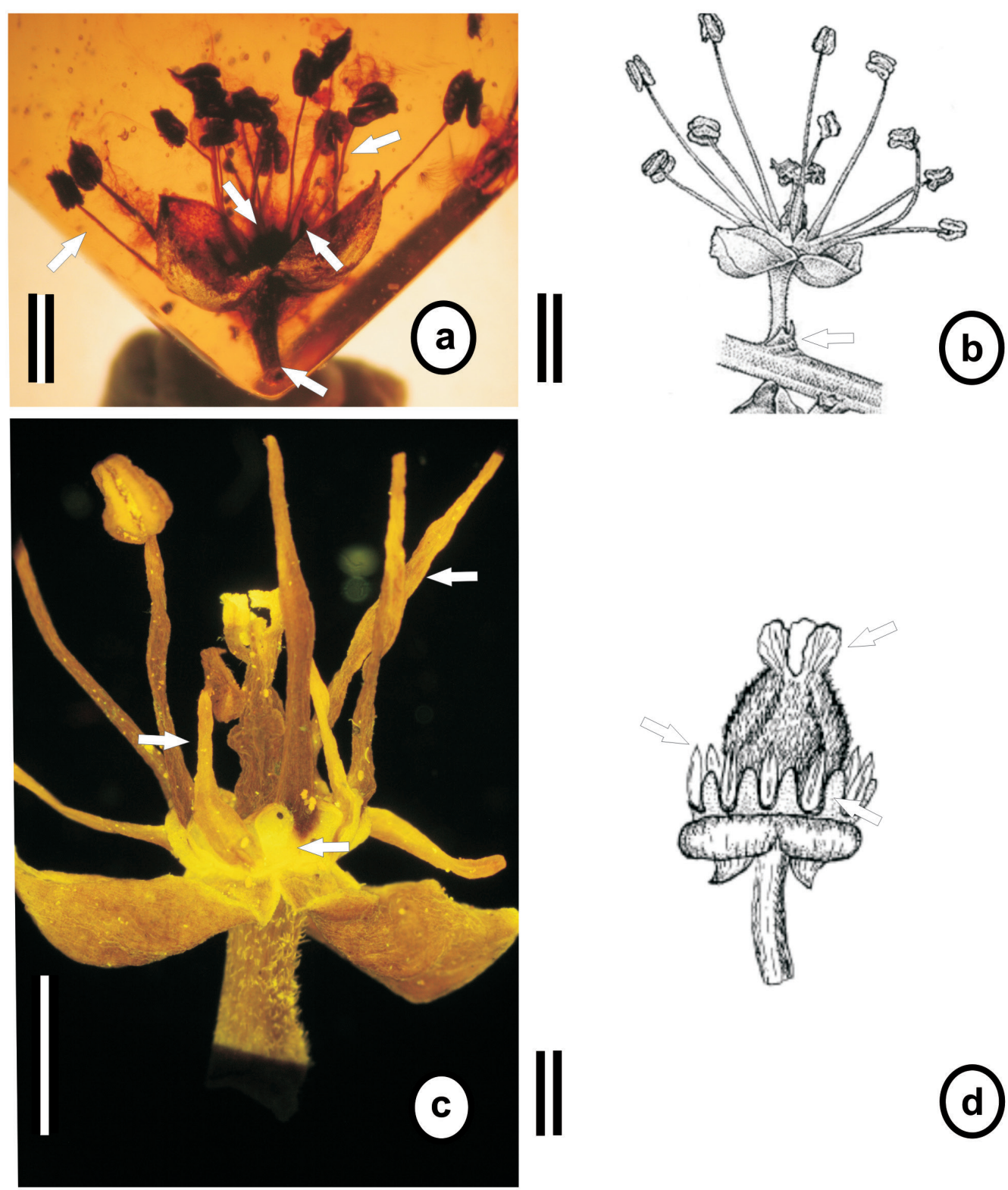

Figura 3. Comparación entre Lunania floresi, L. mexicana y L. parviflora. (a) L. floresi flor bisexual, flecha inferior derecha señalando cicatrices de bractéolas, flecha superior izquierda señalando estambres, flecha media derecha señalando lóbulos del disco, flecha media izquierda señalando ovario súpero con 3 estilos (escala $=1.0 \mathrm{~mm}$ ). (b) L. mexicana. Flecha inferior derecha señalando bractéolas, flecha superior señalando estambres (escala $=1.0$ $\mathrm{mm}$ ) (tomado y modificado de Nee, 1999). (c) L. mexicana flor bisexual, pequeña pedicelada, flecha inferior derecha señalando lóbulos del disco, flecha superior izquierda y derecha señalando dos verticilos de estambres (escala $=0.5 \mathrm{~mm})($ Ejemplar examinado y muestreado: Lunania mexicana Brandegee VERACRUZ: Mpio. San Andrés Tuxtla, Loc. Estación Biológica Tropical Los Tuxtlas. $95^{\circ} 04^{\prime}$ y $95^{\circ} 09^{\prime}$ E y $18^{\circ} 34^{\prime}$ y $18^{\circ} 36^{\prime}$. Lote 67 Alt. 200 msnm Selva alta perennifolia. 13 feb. 1984. G. Ibarra 1257. MEXU:TUX.) (d) L. parviflora, flecha inferior derecha señalando lóbulos del disco, flecha superior izquierda señalando estambres y flecha superior derecha señalando ovario tomentoso y 3 estigmas pequeños (escala $=0.5 \mathrm{~mm}$ ) (tomado y modificado de Robyns, 1968).

representado por la especie Lunania mexicana, la cual crece en selvas altas y medianas de Veracruz, Oaxaca y Chiapas, llegando a Guatemala y Panamá (Nee, 1999; VázquezTorres et al., 2010). La presencia de Lunania floresi, en el Mioceno de Chiapas soporta la idea del desarrollo de una selva tropical durante el tiempo de depósito del ámbar, tal y como lo sugiere el registro fósil de plantas y animales incluidos en el ámbar de Simojovel de Allende (e.g. Miranda, 1963; Poinar y Brown, 2002; Poinar, 2002; Castañeda-Posadas y Cevallos-Ferriz, 2007; CalvilloCanadell et al., 2010; Solórzano-Kraemer, 2010; Chambers et al., 2012), constatando a su vez la larga historia de la integración de la vegetación en México.

La distribución de fósiles de Salicaceae en el tiempo sugiere el desplazamiento del grupo de latitudes altas a latitudes bajas de América del Norte, reforzando así la 
propuesta de la expansión de algunos elementos de la llamada Flora Boreotropical (e.g., Boucher, 2003; CalvilloCanadell, 2010; Collinson, 1992; Gómez-Bravo, 2005; Pérez-Maussán, 2013) a las zonas tropicales de América, especialmente durante el Eoceno-Mioceno. En tiempos más recientes el área de distribución de algunos taxa de ésta flora se amplió a Centro y Sur América. Aunque esta interpretación de la contribución de taxa de latitudes altas de Norte América a la flora y vegetación de la zona tropical de América es atractiva, la influencia de los elementos septentrionales es indudable y el entendimiento de la forma en que plantas de los hemisferios Norte y Sur se sumaron para formar la vegetación actual de la zona Neotropical constituye un tema de álgida discusión, que sólo a través de la suma de estudios detallados se logrará integrar una propuesta más sólida sobre su origen a través del tiempo.

\section{Agradecimientos}

Los autores desean expresar su agradecimiento a la Dra. Hilda Flores Olvera y a la Dra. Helga Ochoterena Booth, del Depto. de Botánica del Instituto de Biología, UNAM, por los comentarios vertidos para el desarrollo de este trabajo, así como al Dr. Guillermo Ibarra Martínez, del Instituto de Investigaciones en Ecosistemas y Sustentabilidad, UNAM, Campus Morelia por facilitar materiales actuales para su revisión. El M. en C. Javier Avendaño Gil quien facilitó la muestra para su estudio. Al Posgrado en Ciencias Biológicas, UNAM y a CONACYT por la beca otorgada (Becario 263534), así como al financiamiento otorgados por los proyectos de CONACYT 221129 y PAPIIT 210416.

\section{Referencias}

Alford, M.H., 2003, Claves para los géneros de Flacourtiaceae de Perú y del Nuevo Mundo: Arnolda, 10, 19-38.

Alford, M.H., 2012, Clave para los géneros de Flacourtiaceae (en línea): Plant Systematics.org, disponible en $<\mathrm{http}$ ://www.plantsystematics. org/index.html $>$, consultado 3 junio 2013.

Alford, M.H., Belyaeva, I.V., 2009, Neotropical Salicaceae (en línea), en Milliken, W., Klitgård, B., Baracat, A., Neotropikey-Interactive key and information resources for flowering plants of the Neotropics, disponible en $<\mathrm{http}$ ://www.kew.org/science/tropamerica/ neotropikey/families/Salicaceae.htm>, consultado 20 mayo 2015.

Avendaño-Gil, J., Carbot-Chacona, G., Coutiño-José, M.A., 2012, Estudio del ámbar con inclusiones biológicas de la Colección Paleontológica de la Secretaría de Medio Ambiente e Historia Natural, Chiapas, México: Lacandonia, 6, 23-29.

Awasthi, N., Srivastava, R., 1989, Some new carbonised Woods from the Neogene of Kerala Coast and their bearing on palaeoclimate: Palaeobotanist, 38, 285-292.

Bande, M.B., 1974, Two fossil Woods from the Deccan Intertrappean beds of Mandla District, Madhya Pradesh: Geophytology, 4, 189-195.

Bande, M.B., Khatri, S.K., 1980, Some more fossil woods from the Deccan Intertrappean beds of Mandla District, Madhya Pradesh, India: Palaeontographica, 173, 147-165.

Bhattacharya, B., 2005, Systematic botany: India, Alpha Science International Limited, $356 \mathrm{p}$.
Berggren, W.A., Van Couvering, J.A.H., 1974, The late Neogene: Palaeogeography, Palaeoclimatology, Palaeoecology, 16, 1-216.

Böse, E., 1905, Geología de Chiapas y Tabasco, Secretaria de Fomento: Instituto Geológico de México, $113 \mathrm{p}$.

Boucher, L.D., Manchester, S.R., Judd, W.S., 2003, An extinct genus of Salicaceae based on twigs with attached flowers, fruits, and foliage from Eocene Green River Formation of Utah and Colorado, USA: American Journal of Botany, 90, 1389-1399.

Calvillo-Canadell, L., Cevallos-Ferriz, S.R.S., Rico-Arce, L., 2010, Miocene Hymenaea flowers preserved in amber from Simojovel de Allende, Chiapas, Mexico: Review of Palaeobotany and Palynology, 160, 126-134.

Castañeda-Posadas, C., Cevallos-Ferriz, S.R.S., 2007, Swietenia (Meliaceae) flower in Late Oligocene-Early Miocene amber from Simojovel de Allende, Chiapas, Mexico: American Journal Botany, 94, 1821-1827.

Centro Estatal de Estudios Municipales (CEEM), 1988, Los municipios de Chiapas. Colección: Enciclopedia de los Municipios de México: Secretaría de Gobernación y gobierno del Estado de Chiapas, México, Documento técnico.

Chambers, K.L., Poinar, G O.Jr., Brown, A.E., 2012, A new fossil species of Colpothrinax (Arecaceae) from Mid-Tertiary Mexican amber: Journal of the Botanical Research Institute Texas, 6, 557-560.

Chandler, M.E.J., 1961, The Lower Tertiary floras of southern England, 1. Paleocene Floras, London Clay Flora (Supplement), Text and Atlas: England, London, British Museum: (Natural History), $354 \mathrm{p}$.

Chase, M.W., Zmarzty, S., Lledo, M.D., Wurdack, K.J., Swensen, S.M., Fay, M., 2002, When in doubt, put it in Flacourtiaceae; a molecular phylogenetic analysis based on plastid rbcl DNA sequences: Kew Bulletin, 57, 141-181.

Collinson, M.E., 1992, The early fossil history of Salicaceae: a brief review: Proceedings of the Royal Society of Edinburgh Section B, 98, 155-167.

Crawley, M., 2001, Angiosperm Woods from British Lower Cretaceous and Palaeogene deposits, Special Papers in Paleontology: London, England, The Paleontological Association, $100 \mathrm{p}$.

Dumortier, B.C.J., 1829, Analyse des Familles de Plantes, avec l'indication des principaux genres qui s'y rattachent, 1-106 $\mathrm{p}$.

Frost, S.H., Langenheim, Jr.R.L., 1974, Cenozoic Reef Biofacies, Tertiary Larger Foraminifera and Scleractian Corals from Chiapas, México: Illinois, U.S.A., Northen Illinois University Press, De Kalb, 388 p.

Gómez-Bravo, A.I., 2005, Estudio taxonómico de una estructura floral conservada en ámbar de Simojovel de Allende, Chiapas, México: Tuxtlas Gutiérrez, Chiapas, Universidad de Ciencias y Artes de Chiapas, tesis de licenciatura, $63 \mathrm{p}$.

Gottwald, H., 1997, Alttertiäre Kieselhölzer aus miozänen Schottern der Ostbayerischen Molasse bei Ortenburg: Documentae Naturae, $109,1-83$.

Greguss, P., 1969, Tertiary angiosperm woods in Hungary: Budapest, Akademiai Kiado, 151 p.

Grimaldi, D., 1996, Amber: window to the past: New York, U.S.A., Publishers and American Museum of Natural History, $216 \mathrm{p}$.

Hooker, W.J., 1844, London Journal of Botany; containing figures and descriptions of such plants as recommend themselves by their novelty, rarity, history, or uses; together with botanical notices and information and occasional memoirs of eminent botanists: London Journal of Botany, 3, 317.

MacGinitie, H.D., 1937, The flora of the Weaverville beds of Trinity County, California with descriptions of plant-bearing beds: U.S.A., Carnegie Institute of Washington Publication, $465 \mathrm{p}$.

Mai, D.H., 1980, Zur Bedeutung von Relikten in der Florengeschichte, en Vent, W., (ed.), Jahre Arboretum 100 (1879-1979), Berlin, 281-307.

Manchester, S.R., 1994, Fruits and seeds of the Middle Eocene Nut Beds flora, Clarno Formation, north central Oregon: Palaeontographica Americana, 58, 1-205.

Manchester, S.R., Dilcher, D.L., Tidwell, W.D., 1986, Interconnected reproductive ad vegetative remains of Populus (Salicaceae) from the Middle Eocene Green River Formation, Northeastern Utah: American Journal of Botany, 73, 156-160. 
Manchester, S.R., Judd, W.S., Handley, B., 2006, Foliage and fruits early poplars (Salicaceae: Populus) from the Eocene of Utah, Colorado, and Wyoming: International Journal of Plant Sciences, 167, 897-908.

Mathiesen, F.J., 1961, On two specimens of fossil wood with adhering bark from the Nugssuaq Peninsula: Meddelelser om Gmnland, 167, 1-54.

Miranda, F., 1963, Two plants from the amber of the Simojovel, Chiapas, Mexico area: Journal of Paleontology, 36, 611-614.

Mirbel, C.F.B. de, 1815, Salicaceae: Éléments de physiologie végétale et de botanique, 2, 905 .

Nee, M., 1999, Flacourtiaceae, Flora de Veracruz, Fascículo 111: Veracruz, Jalapa, Instituto de Ecología, A.C., Universidad de California, Riverside, $79 \mathrm{p}$

Pérez-Maussán, A.I., 2013, Descripción de improntas foliares Eocénicas de la Formación la Carroza, "La Popa”, Nuevo León México, y su comparación con taxa actuales: México, Universidad Nacional Autónoma de México, tesis de licenciatura, 114 p.

Poinar, Jr. G.O., 2002, Fossil palm flowers in Dominican and Mexican amber: Botanical Journal of the Linnean Society, 138 (1), 57-61.

Poinar, Jr. G.O., Brown, A.E., 2002, Hymenaea mexicana sp. nov. (Leguminosae: Caesalpinioideae) from Mexican amber indicates Old World connections: Botanical Journal of the Linnean Society, $139,125-132$.

Prakash, U., Tripathi, P.P., 1972, Fossil woods from the Tertiary of Assam. Palaeobotanist, 21, 305-316.

Ramírez, J.L., Cevallos-Ferriz, S.R.S, 2000, Leaves of Salicaceae (Salix and Populus) from Oligocene sediments, near Tepexi de Rodríguez, Puebla: International Journal of Plant Sciences, 161, 521-534.

Robyns, A., 1968, Flora of Panama. VI: Family 128: Flacourtiaceae: Annals of the Missouri Botanical Garden, 55, 93-144.

Sivarajan, V.V., 1991, Introduction to the principles of Plant Taxonomy: London, England, Cambridge University, 308 p.

Sleumer, H.O., 1980, Flacourtiaceae, en Rogerson, C.T., Flora Neotropica, Monograph No. 22: New York, U.S.A., The New York Botanical Garden, 1-499.

Solórzano-Kraemer, M.M., 2007, Systematic, paleoecology and paleobiogeography of the insect fauna from the Mexican amber: Paleontographica Abteiuung A, 282, 1-33.
Solórzano-Kraemer, M.M., 2010, Mexican amber, en Panney, D. (ed.), Biodiversity of fossil in amber from the major world deposits: Manchester, England, Siri Scientific Press, 42-56.

The Plant List, 2013, ver. 1.1 (en linea), Published on the Internet, disponible en <http://www.theplantlist.org>, consultado $15 \mathrm{de}$ enero de 2015.

Trivedi, B.S., Srivastava, K., 1986, Flacourtioxylon mohgaonense gen. et sp. nov. from the Deccan Intertrappean beds of Mohgaon Kalan, Chhindwara District, M.P. (India): Journal of the Indian Botanical Society, 65, 500-501.

Vázquez-Torres, M., Campos-Jiménez, J., Armenta-Montero, S., CarvajalHernández, C.I., 2010, Árboles de la región de los Tuxtlas: México, Comisión del Estado de Veracruz para la Conmemoración de la Independencia Nacional y la Revolución Mexicana, Veracruz, 377 p.

Vega, F.J., Nyborg, T., Coutaño, M.A., Solé, J., Hernández-Monzón, O., 2009, Neogene Crustacea form sutheastern Mexico: Bulletin of Mizunami Fossil Museum, 35, 51-69.

Wild, H., 1960, Flacourtiaceae, en Exell, A.W., Wild, H. (eds.), Flacourtiaceae (incl.Samydeaceae): Flora Zambesiaca, Mozambique Federation of Rhodesia and Nyasaland Bechuanaland Protectorate: London, Glasgow University Press, 61-298.

Wing, S.L., 1981, A study of the paleoecology and paleobotany in the Willwood Formation (Early Eocene, Wyoming): Connecticut, U.S.A., Yale University, tesis doctoral, 782 p.

Yang Q., Zmarzty, S., 2007, Flacourtiaceae, en Wu, Z., Raven, P.H., (eds.), Flora of China, 13: Beijing, Science Press \& St. Louis, Missouri Botanical Garden, 112-137.

Manuscrito recibido: Septiembre 9, 2015

Manuscrito corregido recibido: Octubre 2, 2015

Manuscrito aceptado: Octubre, 2015 